\title{
ON THE NONEXISTENCE OF GROUPS WITH EXTRA-SPECIAL COMMUTATOR SUBGROUP
}

\author{
MICHAEL D. MILLER
}

\begin{abstract}
In this paper, we extend a result of Joseph and Finkelstein and show that there is no group $G$ such that $G^{\prime}$ is an extra-special $p$-group of exponent $>p(p$ odd $)$.
\end{abstract}

K. Joseph and L. Finkelstein [2] have shown that if $p$ is an odd prime, there does not exist a finite group $G$ satisfying the following three conditions:

(i) $G^{\prime}$ is an extra-special $p$-group of exponent $>p$.

(ii) $Z(G) \subseteq G^{\prime}$.

(iii) $G$ acts irreducibly on $G^{\prime} / Z\left(G^{\prime}\right)$.

It is the object of this paper to prove that their result remains valid even if conditions (ii) and (iii) are dropped. That is, there is no finite group $G$ such that $G^{\prime}$ is an extra-special $p$-group of exponent $>p$ ( $p$ odd $)$.

Recall that a finite $p$-group $G$ is called extra-special if $Z(G)=G^{\prime}$, and $\left|G^{\prime}\right|$ $=p$. We now list a series of lemmas which will be needed for the main theorem. In all that follows, $p$ is an odd prime.

Lemma 1. Let $G$ be an extra-special p-group. Then

(a) $(x y)^{p}=x^{p} y^{p}$

(b) $x^{p} \in Z(G)$ for all $x, y \in G$.

Proof. See [1, p. 183].

LemMA 2. Let $G$ be an extra-special p-group of exponent $>p$, and let $U=\left\{x \in G \mid x^{p}=1\right\}$. Then $U$ is a characteristic subgroup of $G$ and $[G: U]$ $=p$.

Proof. The fact that $U$ is a characteristic subgroup follows immediately from Lemma 1(a), and the fact that automorphisms preserve order. The map $x \rightarrow x^{p}$ is a homomorphism of $G$ onto $Z(G)$ with kernel $U$. Hence $G / U$ $\cong Z(G)$ and $[G: U]=p$.

LEMMA 3. Let $G$ be a finite p-group of linear transformations acting on a vector space $V$ over a field $F$ of characteristic $p$. Then some nonzero vector of $V$ is fixed by every element of $G$.

Proof. See [1, p. 31].

LEMMA 4. Suppose an abelian group $G$ acts as a group of linear transformations

Received by the editors August 15, 1975.

AMS (MOS) subject classifications (1970). Primary 20F35. 
on a vector space $V$ over a field $F$. Let $S$ be a subspace of $V$, and $H$ a subgroup of $G$ whose elements induce scalar transformations on $S$. Let $S^{G}$ be the subspace of $V$ generated by all vectors $s^{g}, s \in S, g \in G$. Then $H$ also induces scalar transformations on $S^{G}$.

Proof. Let $s_{1}^{g_{1}}, s_{2}^{g_{2}} \in S^{G}$, and suppose $h \in H$ with $s^{h}=\lambda s$ for all $s \in S$. Then

$$
\begin{aligned}
\left(s_{1}^{g_{1}}+s_{2}^{g_{2}}\right)^{h} & =s_{1}^{g_{1} h}+s_{2}^{g_{2} h}=\left(s_{1}^{h}\right)^{g_{1}}+\left(s_{2}^{h}\right)^{g_{2}}=\left(\lambda s_{1}\right)^{g_{1}}+\left(\lambda s_{2}\right)^{g_{2}} \\
& =\lambda\left(s_{1}^{g_{1}}+s_{2}^{g_{2}}\right) .
\end{aligned}
$$

Similarly, if $c \in F$, then

$$
\left(c s_{1}^{g_{1}}\right)^{h}=c\left(s_{1}^{g_{1} h}\right)=c\left(s_{1}^{h}\right)^{g_{1}}=\lambda\left(c s_{1}^{g_{1}}\right) .
$$

The lemma follows.

THEOREM. Let $G$ be an extra-special p-group $(p>2)$ of exponent $>p$. Then there is no finite group $K$ such that $K^{\prime}=G$.

Proof. Suppose $K^{\prime}=G$. Then $K$ acts by conjugation on $G^{\prime}$. Moreover, $K$ acts in a natural way on $G / G^{\prime}=\bar{G}$; namely, if $k \in K$, and $a G^{\prime} \in \bar{G}$, then $\left(a G^{\prime}\right)^{k}=\left(k^{-1} a k\right) G^{\prime}$. This is easily seen to be well defined. Now $\tilde{K}=K / G$ is abelian, so we can write $\tilde{K}=\tilde{K}_{p} \times \tilde{K}_{p^{\prime}}$, where $\tilde{K}_{p}$ is a $p$-group, and $\tilde{K}_{p^{\prime}}$ has order prime to $p$. The group $\tilde{K}$ acts in a natural way on $\bar{G}$. Indeed, if $x=k G \in \tilde{K}$, and $a G^{\prime} \in \bar{G}$, then we define $\left(a G^{\prime}\right)^{x}=\left(k^{-1} a k\right) G^{\prime}$. To see that this is well defined, suppose $x=l G$ and $a G^{\prime}=b G^{\prime}$. We need to show that $\left(k^{-1} a k\right) G^{\prime}=\left(l^{-1} b l\right) G^{\prime}$, or equivalently that $l^{-1} b^{-1} l k^{-1} a k \in G^{\prime}$. But

$$
l^{-1} b^{-1} l k^{-1} a k=l^{-1} b^{-1}\left[k l^{-1}, a^{-1}\right]\left(a b^{-1}\right) b l \in G^{\prime},
$$

since $k l^{-1} \in G$, and $a b^{-1} \in G^{\prime}$.

Now $\bar{G}$ is elementary abelian, so it is a vector space over $\mathbf{Z}_{p}$. Let $U=\left\{x \in G \mid x^{p}=1\right\}$, and define $\bar{U}=U / G^{\prime}$. By Lemma $2, U$ has index $p$ in $G$, and $\bar{U}$ is a subspace of $\bar{G}$. As $U$ is characteristic in $G, \bar{U}$ is $\tilde{K}_{p^{\prime}}$-invariant, so by Maschke's Theorem [1, p. 66], there exists a $\tilde{K}_{p^{\prime}}$-invariant subspace $\bar{W} \subseteq \bar{G}$ such that $\bar{G}=\bar{U} \oplus \bar{W}$. Let $\bar{W}=W / G^{\prime}$. As $[G: U]=p, W$ must have order $p^{2}$. Furthermore, $W$ is cyclic since $W \nsubseteq U$.

The action of $\tilde{K}$ on $G^{\prime}$ is given by a character $\lambda: \tilde{K} \rightarrow \mathbf{Z}$; that is, if $k \in \tilde{K}$, then $c^{k}=c^{\lambda(k)}$ for all $c \in G^{\prime}$. Clearly then, $\tilde{K}_{p^{\prime}}$ acts with character $\lambda$ on $G^{\prime}$, hence also on $\bar{W}$. Moreover, $\bar{W}$ is not $\tilde{K}$-invariant. For suppose it were. Then $W$ would be normal in $K$, and $N_{K}(W) / C_{K}(W)=K / C_{K}(W)$ would be abelian, which implies that $W \subseteq Z(G)$, a contradiction. Thus $\bar{W}^{\bar{K}} \cap \bar{U}$ $\neq\{1\}$, where $\bar{W} \bar{K}=\left\langle w^{k} \mid w \in \bar{W}, k \in \tilde{K}\right\rangle$. The $p$-group $\tilde{K}_{p}$ acts on $\bar{W} \tilde{K} \cap \bar{U}$, so by Lemma 3 , there is a subgroup $\bar{V} \subseteq \bar{W}^{K} \cap \bar{U}$, of order $p$, which is elementwise fixed by $\tilde{K}_{p}$.

As $\tilde{K}_{p^{\prime}}$ acts with character $\lambda$ on $\bar{W}$, by Lemma 4 , it acts with character $\lambda$ on $\bar{W}^{K}$, in particular on $\bar{V}$. If $\bar{V}=V / G^{\prime}$, then $V$ is elementary abelian of order $p^{2}$, and since $\bar{V}$ is $\tilde{K}$-invariant, $V \triangleleft K$.

Let $K_{p}$ and $K_{p^{\prime}}$ be defined by $K_{p} / G=\tilde{K}_{p}$ and $K_{p^{\prime}} / G=\tilde{K}_{p^{\prime}}$. Since $\tilde{K}_{p}$ fixes 
$\bar{V}$ elementwise, and $K_{p}$ fixes $G^{\prime}$ elementwise, $K_{p}$ must act on $V$ via matrices of the form $\left(\begin{array}{l}1 \\ * \\ 1\end{array}\right)$. Also, the above discussion shows that $K_{p^{\prime}}$ acts on $V$ via matrices of the form $\left(\begin{array}{c}\lambda \\ * \lambda\end{array}\right)$.

We conclude that $K$ acts on $V$ via an abelian group of matrices, since matrices of the form $\left({ }_{*}^{\mu}\right)$ commute. As $K^{\prime}=G, G$ acts trivially on $V$, that is $V \subseteq Z(G)$. This is a contradiction, and the theorem follows.

It might be worthwhile to note that if a group $G$ is the commutator of any group $K$, then $G$ is also the commutator of a finite group [3]. Hence the theorem is true without the restriction that $K$ be finite.

ACKNowledgments. The author would like to thank Professor D. S. Passman for several valuable conversations.

\section{REFERENCES}

1. D. Gorenstein, Finite groups, Harper \& Row, New York, 1968. MR 38 \#229.

2. L. Finkelstein and $\mathrm{K}$. Joseph, Groups whose commutator subgroup is an extra-special p-group (unpublished).

3. I. D. MacDonald, On cyclic commutator subgroups, J. London Math. Soc. 38 (1963), 419-422. MR 29 \# 143.

4. W. R. Scott, Group theory, Prentice-Hall, Englewood Cliffs, N. J., 1964. MR 29 \#4785.

Department of Mathematics, University of California, Berkeley, California 94720 